\title{
Characterization of the Thickness and Distribution of Latex Coatings on Polyvinylidene Chloride Beads by Backscattered Electron Imaging
}

\author{
Clifford S Todd ${ }^{1}$, Douglas E Beyer ${ }^{2}$ \\ 1. The Dow Chemical Company, Analytical Sciences, Midland, MI USA \\ 2. The Dow Chemical Company, Packaging and Specialty Plastics R\&D, Midland, MI USA
}

Polyvinylidene chloride (PVDC) copolymer resins including SARANTM resins produced by The Dow Chemical Company are commonly used to produce barrier films for food packaging applications [1]. PVDC polymers are particularly advantageous in these applications because they have excellent barrier properties to the flow of oxygen and water vapor over a wide range of environmental conditions. PVDC resins are commonly formulated with a variety of solid additives, including stabilizers, lubricants, extrusion processing aides, colorants, nucleating agents and the like [1]. These are commonly added as small particles in a blending operation. While such a dry blending process is convenient, it does have its drawbacks. In a dry blend the components exist as individual particles, commonly with different particle sizes and/or densities. As a consequence, dry blended formulations are susceptible to segregation of the components during transportation and handling of the resin blend. Such maldistribution of additives can in turn have adverse effects on resin extrusion or film performance. In response, Dow developed a novel process to incorporate solid materials in latex form onto PVDC resin bead surfaces using a coagulation process [2]. This process gives a very uniform distribution of the additive on bead surfaces. Since it is locked onto the surface of the bead it also prevents segregation of the blend components. We developed microscopy techniques in response to the need to characterize the thickness and distribution of these latex coatings on PVDC resin beads.

Using backscattered electron imaging chlorine-bearing material such as PVDC appears brighter than latex, composed predominantly of carbon and hydrogen. The depth reached by backscattered electrons is a function of the accelerating voltage used. As beam electrons enter a sample inelastic scattering events reduce their energy, eventually bringing them to rest. Higher energy electron beams travel deeper than lower energy beams before coming to rest. The depth reached by BSEs follows a similar trend. At a lower voltage of $5 \mathrm{keV}$ the BSE signal comes mostly from the latex on top of the PVDC particle surface. The underlying PVDC can be seen in only a few patches where latex is absent or very thin (Figure 1A). At a higher voltage of $20 \mathrm{keV}$, the beam passes through the layer of latex; the underlying bright PVDC is evident (Figure 1C). The image collected at $10 \mathrm{keV}$ is intermediate between these two; thick latex patches still appear dark, but thinner latex-coated areas appear brighter. Images such as these can be directly compared from sample to sample in order to assess relative differences in latex thickness and coverage.

In order to quantitatively calibrate the BSE image brightness to latex thickness on the PVDC particle surface Monte Carlo simulations were used to model electron-sample interactions [3]. The backscatter coefficient is defined as the fraction of beam electrons that escape the sample as BSEs. Figure 2 shows the backscatter coefficient calculated from the Monte Carlo simulations as a function of accelerating voltage and latex layer thickness. The backscatter coefficient has been normalized between that in pure latex (zero) and pure PVDC (one) at the given accelerating voltage. At each voltage in Figure 2 the trend lines indicate that when the latex coating is thinner the backscatter coefficient is higher (brighter BSE image). The BSE coefficient becomes lower (darker BSE image) when the latex layer is thicker, as 
expected. The latex thickness at which the BSE image becomes dark is a function of accelerating voltage. If a normalized backscatter coefficient of 50\% is chosen as the threshold, for imaging done at 5 $\mathrm{keV}$ a latex layer $100 \mathrm{~nm}$ thick or more would lead to a dark BSE image. Similarly, for $10 \mathrm{keV}$ BSE images, dark areas on beads must have more than 0.35 microns of latex on the surface. For $15 \mathrm{keV}$ BSE images, dark areas have more than 0.75 microns of latex; for $20 \mathrm{keV} \mathrm{BSE} \mathrm{images} \mathrm{dark} \mathrm{areas} \mathrm{have} \mathrm{more}$ than 1.3 microns of latex on the surface. This thickness calibration was validated by cross-sectioning using a focused ion beam SEM.

(B) TMTrademark of The Dow Chemical Company

\section{References:}

[1] BA Howell, and DE Beyer, Encyclopedia of Polymer Science and Technology. DOI:

10.1002/0471440264.pst391.pub2.

[2] SM Kling, Patent US6627679 B1.

[3] D Drouin et al, Scanning 29 (2007) 92-101.

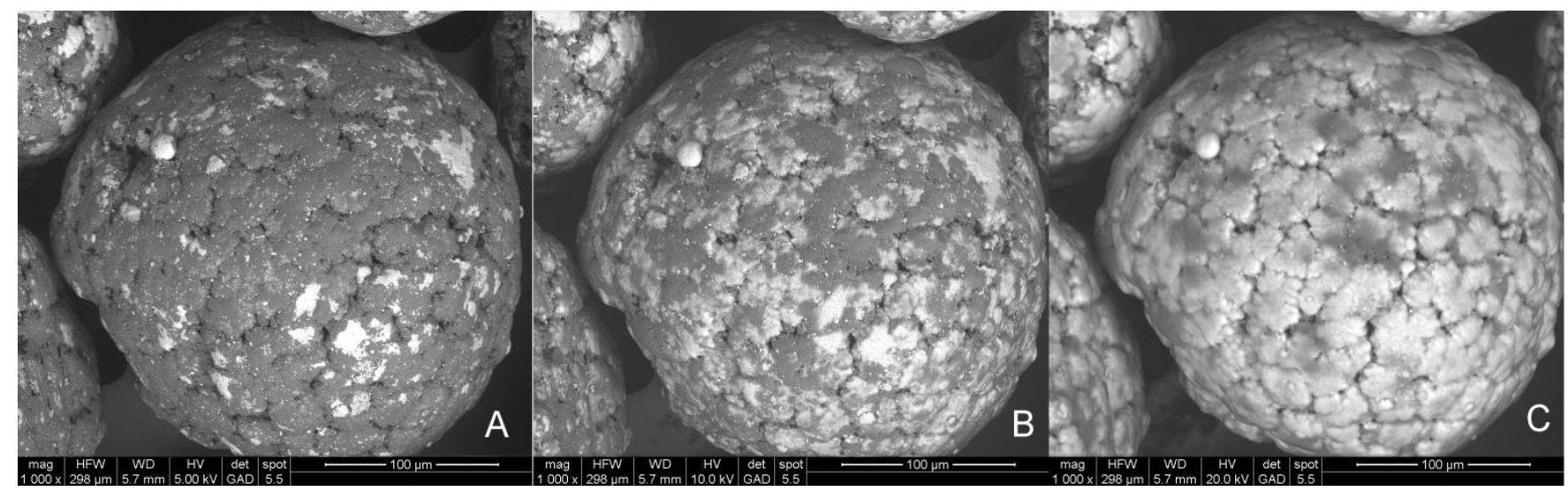

Figure 1. Latex-coted PVDC beads. BSE images of the same field of view at (A) 5, (B) 10 and (C) 20 $\mathrm{keV}$ accelerating voltages. At higher accelerating voltages the BSE signal from the underlying PVDC is detected through progressively thicker latex coatings.

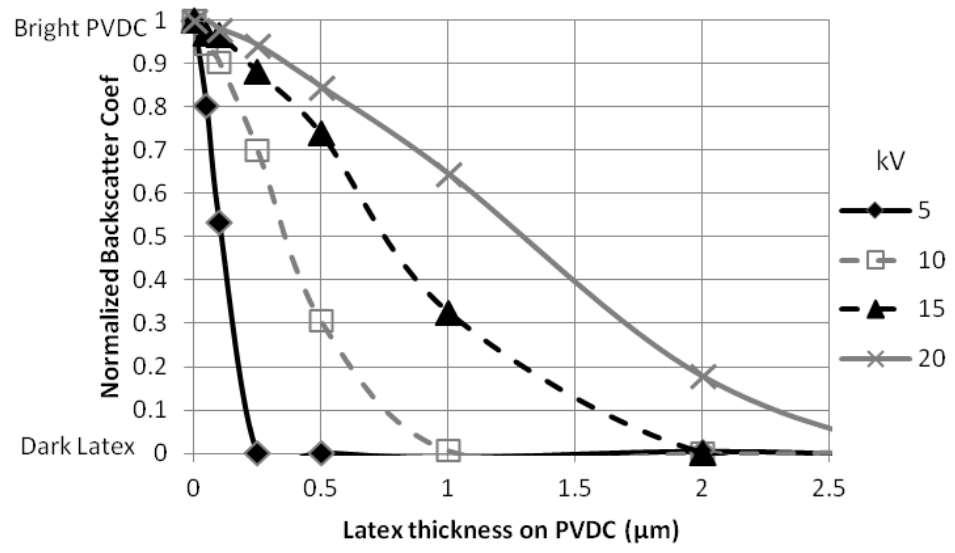

Figure 2. Modeled BSE coefficient as a function of latex layer thickness and accelerating voltage. 